\title{
Accurate template-based modeling in CASP12 using the IntFOLD4-TS, ModFOLD6, and ReFOLD methods
}

Article

Accepted Version

McGuffin, L., Shuid, A. N., Kempster, R., Maghrabi, A. H.A., Nealon, J. O., Salehe, B. R., Atkins, J. D. and Roche, D. B. (2018) Accurate template-based modeling in CASP12 using the IntFOLD4-TS, ModFOLD6, and ReFOLD methods.

Proteins: Structure, Function, and Bioinformatics, 86. pp. 335344. ISSN 0887-3585 doi: https://doi.org/10.1002/prot.25360 Available at https://centaur.reading.ac.uk/71838/

It is advisable to refer to the publisher's version if you intend to cite from the work. See Guidance on citing.

Published version at: http://dx.doi.org/10.1002/prot.25360

To link to this article DOI: http://dx.doi.org/10.1002/prot.25360

Publisher: Wiley

All outputs in CentAUR are protected by Intellectual Property Rights law, including copyright law. Copyright and IPR is retained by the creators or other copyright holders. Terms and conditions for use of this material are defined in the End User Agreement. 


\section{CentAUR}

Central Archive at the University of Reading

Reading's research outputs online 
Title: Accurate Template Based Modelling in CASP12 using the IntFOLD4-TS, ModFOLD6 and ReFOLD methods

Short title: Template Based Modelling (McGuffin Group)

Liam J. McGuffin ${ }^{1}{ }^{*}$, Ahmad N. Shuid ${ }^{1}$, Robert Kempster ${ }^{1}$, Ali H.A. Maghrabi ${ }^{1}$, John O.

Nealon ${ }^{1}$, Bajuna R. Salehe ${ }^{1}$, Jennifer D. Atkins ${ }^{1}$ and Daniel B. Roche ${ }^{2,3}$

1 - School of Biological Sciences, University of Reading, Reading, UK

2 - Institut de Biologie Computationnelle, LIRMM, CNRS-UMR 5506, Université de

Montpellier, Montpellier, France

3 - Centre de Recherche en Biologie cellulaire de Montpellier, CNRS-UMR 5237,

Montpellier, France

* To whom correspondence should be addressed

Liam J. McGuffin

School of Biological Sciences,

University of Reading,

Reading RG6 6AS,

UK

Email: 1.j.mcguffin@ reading.ac.uk

Tel: $+44(0) 1183786332$

Fax: +44 (0)118378 8106

Keywords: Protein Structure Prediction, 3D Modelling, Model Quality Assessment, Refinement, Estimates of Model Accuracy, Accuracy Self Estimate, QA, EMA, ASE

This article has been accepted for publication and undergone full peer review but has not been through the copyediting, typesetting, pagination and proofreading process which may lead to differences between this version and the Version of Record. Please cite this article as an 'Accepted Article', doi: 10.1002/prot.25360

(C) 2017 Wiley Periodicals, Inc.

Received: May 31, 2017; Revised: J ul 12, 2017; Accepted: J ul 25, 2017 


\section{ABSTRACT}

Our aim in CASP12 was to improve our Template Based Modelling (TBM) methods through better model selection, accuracy self-estimate (ASE) scores and refinement. To meet these aims we developed two new automated methods, which we used to score, rank and improve upon the provided server models. Firstly, the ModFOLD6_rank method, for improved global Quality Assessment (QA), model ranking and the detection of local errors. Secondly, the ReFOLD method for fixing errors through iterative QA guided refinement. For our automated predictions we developed the IntFOLD4-TS protocol, which integrates the ModFOLD6_rank method for scoring the multiple-template models that were generated using a number of alternative sequence-structure alignments. Overall, our selection of top models and ASE scores using ModFOLD6_rank was an improvement on our previous approaches. In addition, it was worthwhile attempting to repair the detected errors in the top selected models using ReFOLD, which gave us an overall gain in performance. According to the assessors' formula, the IntFOLD4 server ranked $3^{\text {rd }} / 5^{\text {th }}$ (average Z-score $>0.0 /-2.0$ ) on the server only targets, and our manual predictions (McGuffin group) ranked $1^{\text {st }} / 2^{\text {nd }}$ (average Z-score > 2.0/0.0) compared to all other groups. 


\section{INTRODUCTION}

A principal research focus of our group has been the improvement of methods for Template Based Modelling (TBM) of proteins from their sequences, through accurate Model Quality Assessment (MQA). Improvements in the Estimates of Model Accuracy (EMA) lead to higher quality and more useful 3D models overall, and so the advancement of reliable MQA has always been at the core of our TBM strategy. The development of our IntFOLD tertiary structure (TS) prediction methods ${ }^{1-3}$ stemmed from the development of the GenTHREADER $^{4}$ and nFOLD 5 methods, which integrated several Model Quality Assessment Programs (MQAPs) to improve the recognition of fold templates, initially for the CASP6 experiment. In parallel with nFOLD version 3 at CASP7, we developed the first version of the ModFOLD method, which focused purely on the quality assessment problem ${ }^{6}$. The ModFOLD server method was further improved through integration with clusteringbased variant (ModFOLDclust ${ }^{7}$, which we subsequently used in CASP8, both for our predictions in the QA category ${ }^{8}$ and to rank server models for our manual predictions in the TS category.

In the CASP9 experiment, assessors began to request that predictors include error estimates in Ångströms, in place of the temperature factor (B-factor) field, for each of their submitted 3D models. Since then, the CASP assessors have increasingly placed an emphasis on the importance of "B-factor" predictions, or Accuracy Self Estimate (ASE) scores as they are now termed. The analogy is that relying on a 3D protein model without quality assessment is like trusting the top hit of a BLAST search without knowing the E-value.

The first version of the IntFOLD server ${ }^{9}$ integrated single template modelling with ModFOLDclust2 for ranking and ASE scores, which were included in the "B-factor" column of the model output files. In CASP9, the IntFOLD-TS method ${ }^{1}$ gained attention for its high 
performance in the assignment of model reliability/"B-factors", which was first evaluated in the TBM category ${ }^{10}$.

The original IntFOLD server only used a single-template modelling approach and so with IntFOLD2-TS, for CASP10, our aim was to exploit our strengths in ASE scoring and use the scores to guide our multiple-template modelling protocols ${ }^{2}$. For CASP11, with our IntFOLD3-TS method ${ }^{3}$ we added some extra sequence-structure alignment methods, but we used the same multiple-template modelling ranking and ASE scoring protocols that were used by IntFOLD2.

For our manual TS predictions in CASP8-CASP11 we used ModFOLD variants for ranking server models and adding ASE scores to our submitted models. We had some success with high cumulative GDT_TS rankings, but historically our main strength in the TS prediction category has been with our ASE scores. Until CASP12, we had always been able to accurately identify errors in the server models but we had not developed any reliable methods for fixing them.

In this paper, we describe our latest improvements to the IntFOLD-TS method (Version 4), which integrates the new ModFOLD6 ${ }^{11}$ variant, ModFOLD6_rank, for improved selection and ASE scoring. Additionally, in CASP12 for the first time we have made serious efforts at model refinement guided by ASE scores using our new ReFOLD ${ }^{12}$ method.

\section{METHODS}

\section{The IntFOLD4-TS prediction server}

For CASP12, a bespoke version of the IntFOLD4 server was developed in order to return appropriately formatted results for the tertiary structure (TS) prediction category. Additionally, the local quality assessment predictions (ASE scores) were returned as 
predicted distances in the B-factor column of each TS model file using our ModFOLD6 ${ }^{11}$ QA server (N.B. predictions in the EMA/QA category were also returned by our ModFOLD6 and ModFOLDclust2 servers, see our EMA methods paper in this issue for more details).

Our IntFOLD4-TS method was developed with the aim of identifying, and then attempting to fix, the local errors in an initial pool of single template models via iterative multi-template modeling. The method attempts to exploit our previous CASP successes in accurately predicting local errors in our models ${ }^{1}$ by taking the global and local per-residue errors into consideration during the multiple template selection stage ${ }^{2}$.The pipeline can be broken down into two major stages: i) single template modelling with ASE scoring and ii) QA guided multiple template modelling with ASE scoring (Figure 1).

For the single template modelling first major stage, 14 different fold recognition methods were installed and run in-house, generating up to 10 sequence-to-structure alignments each; this resulted in up to 140 alternative single-template based models being generated for each CASP target. The following fold recognition methods were used: SP $3^{13}$, SPARKS2 ${ }^{13}$, HHsearch $^{14}, \mathrm{COMA}^{15}, \mathrm{SPARKSX}^{16}, \mathrm{CNFsearch}^{17}$ and the 8 alternative threading methods that are integrated into the current LOMETS package ${ }^{18}$ (PPA, dPPA, dPPA2, sPPA, MUSTER, wPPA, wdPPA and wMUSTER). At the end of the first stage, all single-template models were assessed using ModFOLDclust $2^{19}$ in order to assign global and local model quality scores.

In the second major stage, the single-template model quality scores, and other criteria involving template coverage, were used to select sequence-structure alignments for building multiple-template models ${ }^{2}$. The overall aim was to select appropriate target-template alignments that would minimise local errors in the final models. The Multiple Template Modelling (MTM) stage included 4 main alternative alignment selection methods (multi1multi4) for 3D model building. The first method, multi1, simply used the top 2 alignments 
according to the template ranking. The multi2 method used the top ranked alignment and any subsequent alignments if there were $>=40$ new residues covered and $<=20$ residues were overlapping. The multi3 method used the top ranked alignment and any subsequent alignments, but only if the overlapping region was predicted to increase local model quality. Finally, the multi4 method used the top ranked alignment and any subsequent alignments, but only if the coverage was increased by at least 1 residue. Four additional variants on these methods (multi5-multi8) repeated multi1-multi4, respectively, however, the alignments for each of the single-template methods were firstly re-ranked based on the ModFOLDclust2 predicted global model quality scores. These MTM approaches were first introduced in our IntFOLD2-TS method and they are fully described and benchmarked in our paper published in $2012^{2}$.

The alternative MTM alignment selection methods resulted in the generation of a new population of up to 124 multi-template models for each target. Additionally, ITASSER_LIGHT ${ }^{20}$ (I-TASSER4.4 run in "light mode" with wall-time restricted to 5h; for sequences <600 residues) and HHpred ${ }^{21}$ were used to generate 3 models each, which were then added into the final pool of alternative multi-template models for ranking. In the final stage of the method, the $\sim 130$ models in the final reference set were then evaluated using our ModFOLD6_rank ${ }^{11}$ QA method and the top 5 ranked models were submitted as the final IntFOLD4-TS predictions (see also our EMA paper in this issue for more details about our ModFOLD6_rank method).

\section{The McGuffin group TS predictions}

Our manual TS prediction protocol is shown in Figure 2. Initially, for each target, the server models were ranked according to their ModFOLD6_rank global quality scores. The top ranked initial model was then selected and submitted to the ReFOLD pipeline, which has 
now been implemented as a server ${ }^{12}$. The ReFOLD method consisted of three protocols. The first protocol used a rapid iterative strategy (i3Drefine ${ }^{23}$ ) and the second protocol employed a more CPU/GPU intensive molecular dynamic simulation strategy (using NAMD ${ }^{24}$ ) to refine each starting model. Refined models generated from each protocol were assessed and ranked using ModFOLD6_rank. The third protocol was a combination of the first 2 approaches, where the top ranked model from the 2nd protocol was further refined using i3Drefine. Finally, all of the refined models generated by each of these protocols and the starting model were pooled and re-ranked again using ModFOLD6_rank and the final top 5 models were selected and submitted. For each model, the ModFOLD6 predicted per-residue error scores were added into the B-factor column for each set of atom records

In addition to our independent submissions, we were also the submitters for the collaborative WeFOLD group, wfRosetta-ProQ-ModF6, which also made use of ModFOLD6_rank for final model ranking and ASE scoring (paper in preparation).

\section{Benchmarking methods prior to CASP12}

Prior to CASP12, we benchmarked our ModFOLD6 methods against our existing methods to gauge their ability to rank CASP11 server models and their ASE scoring capability. We then built the ModFOLD6_rank method into the IntFOLD4 server and continuously benchmarked both the IntFOLD4-TS and ModFOLD6 servers using the independent CAMEO resource ${ }^{25}$.

\section{RESULTS}

\section{Benchmarking prior to CASP12}

Prior to CASP, our main priority was to benchmark our new methods to confirm that they were working as intended for model selection, and importantly, outperforming our older 
server TS methods (IntFOLD2-TS ${ }^{2}$ and IntFOLD3-TS ${ }^{3}$ ) and QA methods (ModFOLD4 ${ }^{26}$ and ModFOLD5). In CASP11, we used the IntFOLD3-TS method for our server TS predictions and ModFOLD5 (which was similar in performance to ModFOLD4), in order to select the top server models from our manual submissions.

Figure 3 shows our in-house benchmarking of the ModFOLD6_rank method compared with other QA methods for model selection using CASP11 data. The results show that the ModFOLD6_rank method obtains higher cumulative GDT_TS scores for model selection $\left(\sum \mathrm{GDT}=44.42\right)$ than its component methods ${ }^{11,27}$ as well as outperforming the older ModFOLD5_single and ModFOLDclust2 methods ( $\sum$ GDT_TS=40.06 and $\sum$ GDT_TS=42.68 , respectively), which were used in CASP11 for QA and model selection.

The ModFOLD6_rank method was built into the IntFOLD4-TS pipeline and then benchmarked against our previous servers using the CAMEO resource ${ }^{25}$. The direct comparison of the performance of the IntFOLD4-TS server versus the other servers is shown in Table 1. According to the CAMEO-3D results, using 12 months of data and a common subset of 500 targets, IntFOLD4-TS is shown to outperform our own older servers (IntFOLD2-TS \& IntFOLD3-TS), and all but one other server - Robetta (a direct 12 month comparison of IntFOLD4-TS and Robetta on more targets is shown in Table S1). The CAMEO analysis using 6 months of data shows the same ranking of servers (Table S2). In addition to benchmarking IntFOLD4-TS with CAMEO-3D, the ModFOLD6 server is continually benchmarked with CAMEO-QE in terms of ASE/local score predictions, and is verified to outperform our older method, ModFOLD4, as well as most others.

\section{CASP12 results - TBM and TBM/FM performance comparison with other groups}

Tables 2 and 3 show the relative performance of our group methods versus other top 20 groups on the manual and server-only targets respectively. Table 2 shows that our manual prediction group (McGuffin) is ranked as the top group by SUM Zscore $(>0.0)$ and AVG 
Zscore (>0.0) and 2nd top by other rankings. Our WeFOLD group (wfRosetta-ProQ-ModF6; paper in preparation), which used ModFOLD6_rank for final model selection and ASE scoring, is also shown to be best out of the WeFOLD groups by all rankings, the 5 th best group overall by AVG Zscore (>0.0), and within the top 10 overall for all score rankings.

On the 'all groups' targets, our IntFOLD4 server is competitive with the manual groups, ranking as the 11th best group overall by SUM Zscore (>-2.0) and the 3rd best server group by all rankings (Table 2). The relative performance of IntFOLD4 is also reflected on the 'server only' targets where it ranks 3rd best by SUM Z-score (>-2.0) and AVG Z-score (>-2.0) and 5th by other rankings (Table 3).

\section{CASP12 results - comparison of top models produced at different stages of the IntFOLD4-TS pipeline}

One of the questions asked at the CASP12 meeting was: "Which stages of the IntFOLD4-TS pipeline are worthwhile and actually show improvements?" The results in Table 4 and supplementary Tables S3-S9 show our in-house comparison of the IntFOLD4-TS performance versus the single-template modelling stages and reference multiple-template modelling methods. The cumulative scores of the top models from the IntFOLD140 singletemplate stage, which uses models from all 14 sequence-structure alignment methods, is shown to outperform the other single-template model ranking stages, which use fewer input alignments (IntFOLD60 and LOMETS4.4). This result indicates that adding more alignment methods leads to higher performance on average, according to the GDT-TS scores (Table 4, Table S6), GDT_HA scores (Tables S3 \& S7), TMscores (Tables S5 \& S8) and MaxSub scores (Tables S5 \& S9).

Furthermore, the cumulative scores from the single-template methods are shown to be outperformed by those of the multiple-template modelling methods, indicating that using multi-templates for building models is a worthwhile stage of the pipeline. In addition, the 
models from IntFOLD4-TS are of higher quality overall than those from the other multipletemplate methods (IntFOLD3-TS, HHpred and I-TASSER_LIGHT) according to all scores. The pairwise t-tests results show that the IntFOLD4 top models are of significantly higher quality than those from each of the single-template model ranking stages, according to all scores. In comparsion with the multi-template methods, the pairwise t-tests on the data in Table 4 and supplementary Tables S1-S7 do not provide evidence that the improvement in scores over those from HHpred is statistically significant, however the scores are shown to be significantly better than those from the I-TASSER_LIGHT and IntFOLD3-TS methods.

\section{DISCUSSION}

For our CASP12 TS predictions, our main aims were to: i) increase the diversity of models, using more sequence-structure alignment methods, ii) improve the ranking of models and increase the quality of the top selected models, iii) improve detection of local errors in models (ASE scores) and finally, iv) fix the detected errors in the models.

\section{What we did differently in CASP12 compared with CASP11}

We developed 3 new methods to help us meet our aims: i) the IntFOLD4 server (group number 405) for TS predictions, ii) the ModFOLD6 variants (groups 201, 072, 360) for model ranking/selection and ASE, and iii) the ReFOLD method for repairing models using QA guided iterative refinement (N.B. ReFOLD was used as a standalone in-house tool during CASP12, but is now a publicly available server).

For our IntFOLD4 server TS predictions, the major improvement over the IntFOLD3TS method (used in CASP11) was the integration of our new ModFOLD6_rank model quality assessment method for final model selection and ASE, instead of using ModFOLDclust2. Additionally, the sequence-structure alignment method CNFsearch was 
added to the initial single-template modelling stage and we added HHpred models to the final ranking stage. The ReFOLD method was not incorporated into the IntFOLD4-TS server pipeline, as it was not fully automated during the prediction season.

For our manual predictions (McGuffin, group 017), we also used the ModFOLD6_rank method, for model selection and ASE scoring, but in addition, we used our ReFOLD iterative refinement method, which was guided by the model quality scores. Conversely, in CASP11 we only used the ModFOLDclust2 method for server model selection and ASE scores and we did not carry out any refinement.

\section{What went right?}

Our IntFOLD4-TS server is a significant improvement over our previous automated approach, IntFOLD3-TS (Table4 \& Tables S1-S7), and this result is reflected in the independent CAMEO benchmark results. At the time of writing, IntFOLD4 ranks as the 2nd best 3D server according to the CAMEO IDDT scores based on pairwise comparisons (it is outperformed by only 1 public server in the benchmark - Robetta). Our ModFOLD6_rank method significantly improves upon our previous approaches for quality assessment, particularly for top model selection (Figure 3 ) and for providing local error estimates ${ }^{11}$. On average, the ReFOLD method provided a further significant improvement in model quality of our submitted modes for the regular (T0) $\operatorname{targets}^{12}$. Figure 4A \& B show two examples of TBM/FM targets where we successfully selected from among the best server models and then further improved them with refinement. On average, mostly things went right - overall our selection of top models and ASE scoring was successful and it was worthwhile attempting to repair the errors with ReFOLD.

\section{What went wrong?}


If CASP12 groups are ranked on the TBM \& TBM/FM targets using GDT_TS based metrics alone, then our methods are no longer at the very top; the McGuffin group rankings drop from $1^{\text {st }} / 2^{\text {nd }}$ place to $5^{\text {th }}$ place overall, and the IntFOLD4 server drops from $3^{\text {rd }} / 5^{\text {th }}$ to $9^{\text {th }} / 10^{\text {th }}$ place on the server only targets (http://predictioncenter.org/casp12/zscores_final.cgi). We clearly got an extra ranking boost from our ASE scores and we are grateful that the assessors placed an emphasis on it in their scoring formula.

Despite our significant progress with ModFOLD6_rank ${ }^{11}$, the method is still sub optimal in terms of model ranking, which led to some poorly chosen IntFOLD4 final models and initial server models used by the McGuffin group. Additionally, the IntFOLD4 server is outperformed on the FM targets by many methods, and presently, the server does not integrate our refinement protocol (http://predictioncenter.org/casp12/zscores_final.cgi).

Our refinement of 3D models is still a bit hit and miss; although we have improved upon our identification of errors (we know which parts of a model are likely to be incorrect), we still can't consistently repair them. The current ReFOLD approach is very inconsistent on easier targets with higher quality starting models, as there is less room for improvement and detecting smaller changes in quality is more difficult ${ }^{12}$. Figure $4 \mathrm{C}$ shows an example where our initial model selection was sub optimal and then our refinement process made the model even worse. Figure 4D shows an example where we managed to successfully refine the starting model, however we should have selected our own server model instead, which had a higher GDT_TS score.

\section{CONCLUSIONS}

\section{What we learned and our future plans}


Focusing on model selection, refinement and maximising ASE scoring is clearly a worthwhile strategy. The emphasis by the assessors on the ASE performance is clearly very important. A model (or parts of it) could be very unreliable, even if it is from a good method, in which case, it could be worse than useless if biologists rely upon it to inform their experiments. Biologists are therefore becoming increasingly sophisticated in their use of 3D protein models and we have observed a steady growth in citations and users of our model quality assessment servers ${ }^{11}$. The major contribution to our group performance came from the ModFOLD6_rank method, i.e., improved model selection and ASE scoring. Additionally, we gained a small overall performance boost in GDT_TS by attempting refinement for the T0 targets using ReFOLD ${ }^{12}$.

We will continue to work on improving our model ranking, selection and ASE scoring with future versions of ModFOLD. With ReFOLD, we will work on detecting smaller changes in improvements to models (for good quality starting models). In addition, we will work on a more focused ReFOLD protocol, which makes better use of our strengths in ASE scoring, by concentrating on improving only the low quality residues in the starting models. The future versions of the ModFOLD and ReFOLD methods will be integrated with later versions of the IntFOLD server. For our manual predictions, we plan to continue to make use of the server models, and likewise, we will use future versions ModFOLD for selection and ReFOLD for refinement.

\section{FUNDING}

This work was supported by studentships from: the Malaysian Government (to A.N.S.), the Saudi Arabian Government (to A.H.A.M.), the Tanzanian Government through the Institute of Finance Management (IFM) (to B.R.S.), and the University of Reading and the Diamond 
Light Source Ltd. (to J.D.A.). This work was also supported by IBC ANR Investissements D'Avenir (to D.B.R.).

\section{REFERENCES}

1. McGuffin LJ, Roche DB. Automated tertiary structure prediction with accurate local model quality assessment using the IntFOLD-TS method. Proteins 2011;79 Suppl 10:137146.

2. Buenavista MT, Roche DB, McGuffin LJ. Improvement of 3D protein models using multiple templates guided by single-template model quality assessment. Bioinformatics 2012;28(14):1851-1857.

3. McGuffin LJ, Atkins JD, Salehe BR, Shuid AN, Roche DB. IntFOLD: an integrated server for modelling protein structures and functions from amino acid sequences. Nucleic Acids Res 2015;43(W1):W169-173.

4. McGuffin LJ, Jones DT. Improvement of the GenTHREADER method for genomic fold recognition. Bioinformatics 2003;19(7):874-881.

5. Jones DT, Bryson K, Coleman A, McGuffin LJ, Sadowski MI, Sodhi JS, Ward JJ. Prediction of novel and analogous folds using fragment assembly and fold recognition. Proteins-Structure Function and Bioinformatics 2005;61:143-151.

6. McGuffin LJ. Benchmarking consensus model quality assessment for protein fold recognition. Bmc Bioinformatics 2007;8:345.

7. McGuffin LJ. The ModFOLD server for the quality assessment of protein structural models. Bioinformatics 2008;24(4):586-587.

8. McGuffin LJ. Prediction of global and local model quality in CASP8 using the ModFOLD server. Proteins 2009;77 Suppl 9:185-190.

9. Roche DB, Buenavista MT, Tetchner SJ, McGuffin LJ. The IntFOLD server: an integrated web resource for protein fold recognition, 3D model quality assessment, intrinsic disorder prediction, domain prediction and ligand binding site prediction. Nucleic Acids Res 2011;39(Web Server issue):W171-176.

10. Mariani V, Kiefer F, Schmidt T, Haas J, Schwede T. Assessment of template based protein structure predictions in CASP9. Proteins 2011;79 Suppl 10:37-58.

11. Maghrabi AHA, McGuffin LJ. ModFOLD6: an accurate web server for the global and local quality estimation of 3D protein models. Nucleic Acids Res 2017.

12. Shuid AN, Kempster R, McGuffin LJ. ReFOLD: a server for the refinement of 3D protein models guided by accurate quality estimates. Nucleic Acids Res 2017.

13. Zhou H, Zhou Y. SPARKS 2 and SP3 servers in CASP6. Proteins 2005;61 Suppl 7:152-156.

14. Soding J. Protein homology detection by HMM-HMM comparison. Bioinformatics 2005;21(7):951-960. 
15. Margelevicius M, Venclovas C. Detection of distant evolutionary relationships between protein families using theory of sequence profile-profile comparison. Bmc Bioinformatics 2010;11.

16. Yang YD, Faraggi E, Zhao HY, Zhou YQ. Improving protein fold recognition and template-based modeling by employing probabilistic-based matching between predicted onedimensional structural properties of query and corresponding native properties of templates. Bioinformatics 2011;27(15):2076-2082.

17. Ma JZ, Wang S, Zhao F, Xu JB. Protein threading using context-specific alignment potential. Bioinformatics 2013;29(13):257-265.

18. Wu ST, Zhang Y. LOMETS: A local meta-threading-server for protein structure prediction. Nucleic Acids Res 2007;35(10):3375-3382.

19. McGuffin LJ, Roche DB. Rapid model quality assessment for protein structure predictions using the comparison of multiple models without structural alignments.

Bioinformatics 2010;26(2):182-188.

20. Roy A, Kucukural A, Zhang Y. I-TASSER: a unified platform for automated protein structure and function prediction. Nat Protoc 2010;5(4):725-738.

21. Meier A, Soding J. Automatic Prediction of Protein 3D Structures by Probabilistic Multi-template Homology Modeling. Plos Comput Biol 2015;11(10).

22. Marti-Renom MA, Stuart AC, Fiser A, Sanchez R, Melo F, Sali A. Comparative protein structure modeling of genes and genomes. Annu Rev Bioph Biom 2000;29:291-325. 23. Bhattacharya D, Nowotny J, Cao R, Cheng J. 3Drefine: an interactive web server for efficient protein structure refinement. Nucleic Acids Res 2016;44(W1):W406-409.

24. Phillips JC, Braun R, Wang W, Gumbart J, Tajkhorshid E, Villa E, Chipot C, Skeel RD, Kale L, Schulten K. Scalable molecular dynamics with NAMD. J Comput Chem 2005;26(16):1781-1802.

25. Haas J, Roth S, Arnold K, Kiefer F, Schmidt T, Bordoli L, Schwede T. The Protein Model Portal--a comprehensive resource for protein structure and model information. Database (Oxford) 2013;2013:bat031.

26. McGuffin LJ, Buenavista MT, Roche DB. The ModFOLD4 server for the quality assessment of 3D protein models. Nucleic Acids Res 2013;41(Web Server issue):W368-372. 27. Uziela K, Wallner B. ProQ2: estimation of model accuracy implemented in Rosetta. Bioinformatics 2016;32(9):1411-1413. 


\section{FIGURE LEGENDS}

Figure 1. Flowchart outlining the principal stages of stages of the IntFOLD4-TS prediction pipeline. Rectangles show processes, parallelograms show datasets. The only input is the target sequence. The initial single-template modelling stages start with 14 sequence-structure alignment methods ( 8 from the LOMETS ${ }^{18}$ package $\& 6$ others as described in the main text $\left.{ }^{13-17}\right)$. Single-template models are built from the various alignment methods using MODELLER $^{22}$ (creating the IntFOLD60, IntFOLD140 model datasets) and then ranked with ModFOLDclust $2{ }^{19}$. LOMETS4.4 is also used to rank the backbone models produced by its own component threading methods. The multiple-template modelling stages include QA guided multi-template modelling (using the scores from ModFOLDclust2) in order to generate a set of multi-template models. Additionally, models from $\mathrm{HHpred}^{21}$ and ITASSER_LIGHT ${ }^{20}$ are added to the final IntFOLD4_multi set for evaluation. The ModFOLD6_rank method ${ }^{11}$ is used for ASE and final model selection.

Figure 2. Flow of data and methods used by the McGuffin group for making TS predictions. The initial server models for each target were ranked using the ModFOLD6_rank method. The top ranked server model was then refined using ReFOLD to produce a set of alternatives, which were then further scored and ranked against the original top model using ModFOLD6_rank.

Figure 3. Benchmarking the performance of QA methods for model selection using CASP11 data, prior to CASP12. ModFOLD6_rank versus other global scoring methods: SSA, Secondary Structure Agreement; DBA, Disorder B-factor Agreement; CDA, Contact Distance Agreement ${ }^{11}$. Cumulative GDT scores for the top selected models from the QA targets (models from QA round1 and round2 combined, 84 targets with structure). The 
maximum possible GDT_TS (MaxGDT_TS) is the cumulative score obtained by selecting the best model available for every target. The error bars show the Standard Error in GDT_TS $(\sigma / \sqrt{ } \mathrm{n}$, where $\sigma$ is the standard deviation and $\mathrm{n}$ is the number of targets (84)).

Figure 4. Examples of what went right $(\mathbf{A} \& \mathbf{B})$ and what went wrong $(\mathbf{C} \& \mathbf{D})$ with our manual TBM \& TBM/FM predictions. Left panels, refined model with the ModFOLD6 accuracy self-estimate (ASE) displayed using the temperature colour scheme (blue $=$ residues close to the native structure, red= residues far from the native structure). Middle panels, superposition of the top selected server model (cyan), refined model (magenta) and native structure (green). Right panels, GDT plots comparing top selected server models (cyan) with the ReFOLD refined models (magenta). (A) T0912TS017_1-D2 (McGuffin TS1) - Perfect initial model selection (best model $=$ GOAL_TS1), ASE score $=82.28$, and successful refinement with an improvement on the initial model (GDT_TS from 62.95 to 65.36) (B) T0892TS017_1-D1 (McGuffin TS1) - Excellent initial model selection (2nd best server model = ZhangServer_TS3), ASE score $=80.95$, and successful refinement (GDT_TS improved from 79.71 to 82.25). (C) T0942TS017_1-D1 (McGuffin TS1) suboptimal initial model selection (QUARK_TS1), ASE score $=86.09$, and unsuccessful refinement $($ GDT_TS declined from 79.91 to 79.33). (D) T0896TS017_1-D2 (McGuffin TS1) ASE score $=81.45$, refinement successful (GDT_TS improved from 42.12 top 45.25), but suboptimal initial model selection (GOAL_TS2), our own IntFOLD4 TS1 model was better overall (GDT_TS=46.50). 
ע

Target sequence

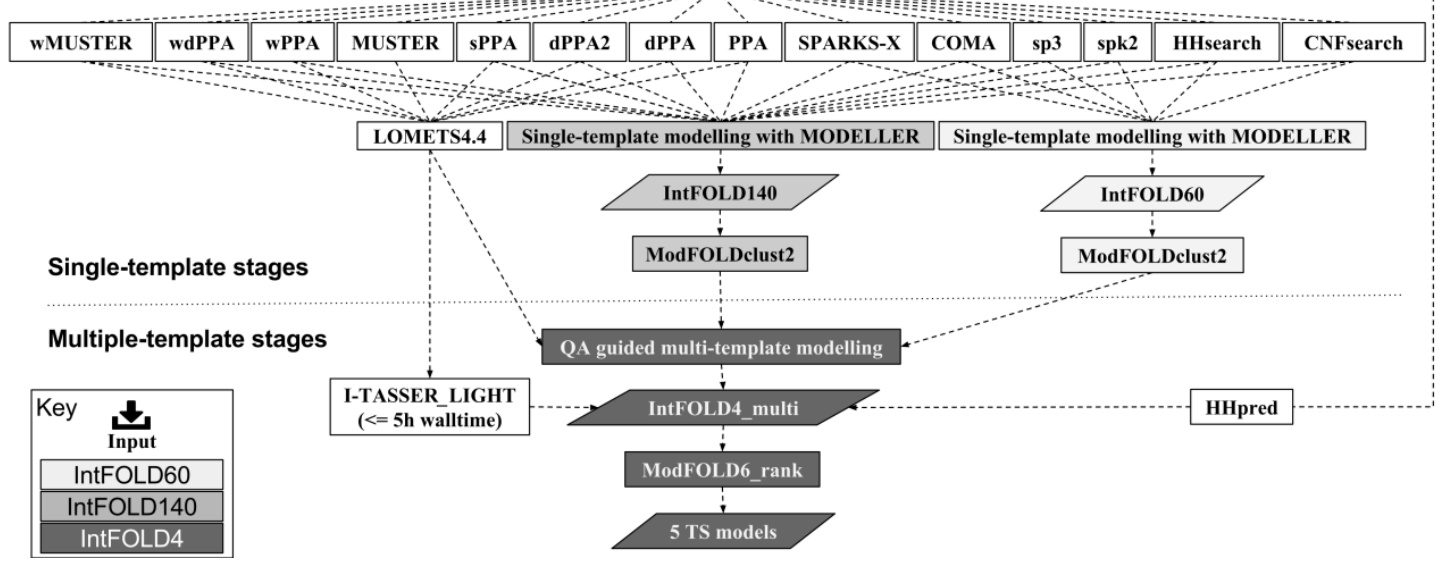

Target sequence $\rightarrow$

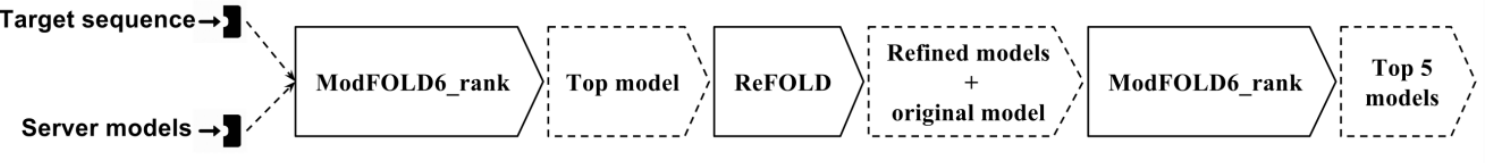

\begin{tabular}{c} 
Key \\
\multicolumn{1}{c|}{ Input $\rightarrow$; } \\
\hline Data \\
\hline Method \\
\hline
\end{tabular}



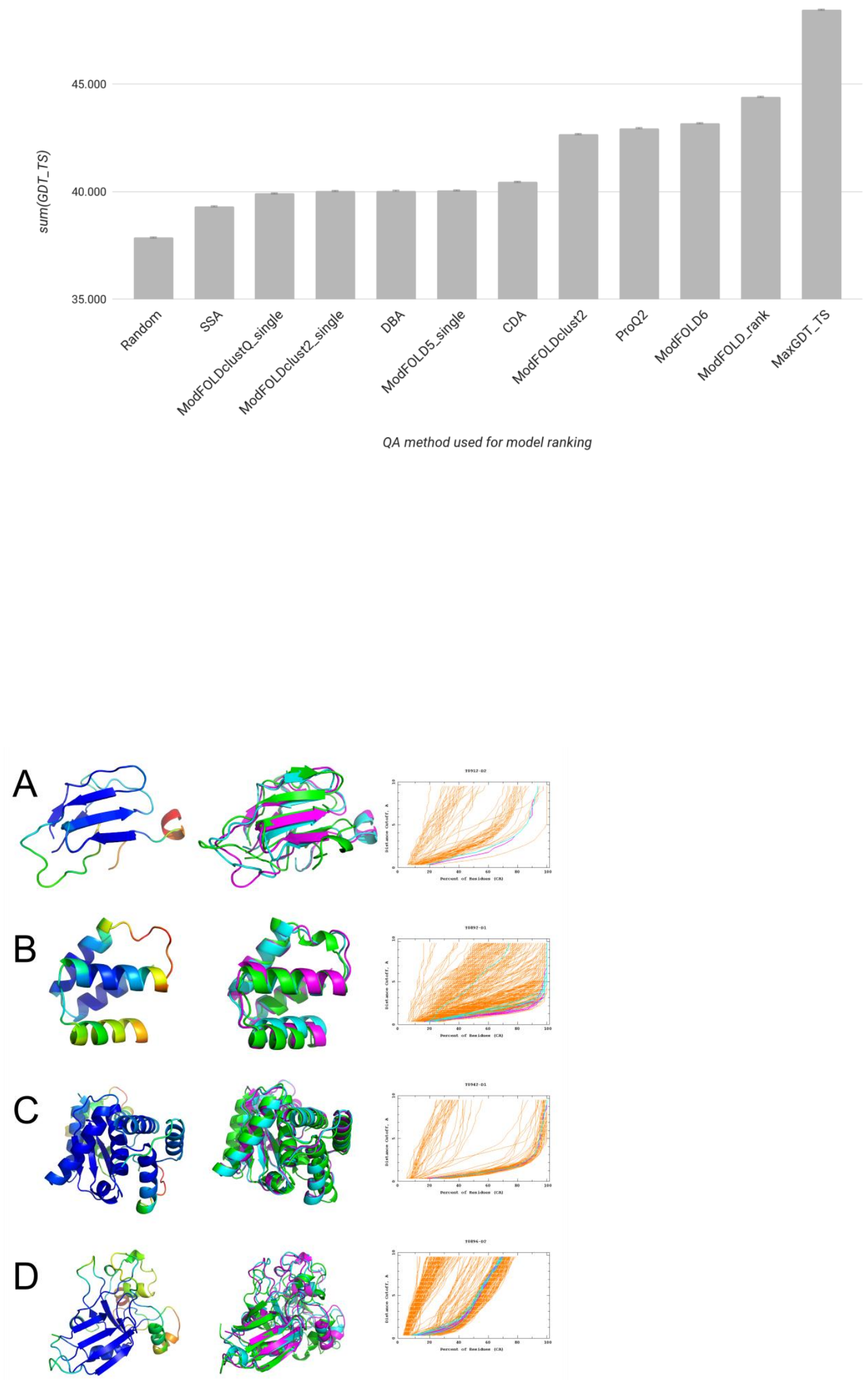
Table 1. Performance of IntFOLD4-TS versus other servers. CAMEO-3D: Common Subset Comparison, 1-year Performance (2016-05-13 - 2017-05-06) (500 targets - 10 methods). IntFOLD4_TS is the reference server (listed as server58, or IntFOLD4-TSb on CAMEO). Data are from http://www.cameo3d.org/. The table is sorted by difference in Average IDDT score.

\begin{tabular}{|l|r|r|r|r|r|r|}
\hline & \multicolumn{2}{|c|}{ Average IDDT } & \multicolumn{2}{l|}{ Average CAD score } & \multicolumn{2}{l|}{ Average IDDT-BS } \\
\hline \multicolumn{1}{|c|}{ Server Name } & \multicolumn{1}{c|}{ Dif. } & \multicolumn{1}{c|}{ Ref. } & \multicolumn{1}{c|}{ Dif. } & \multicolumn{1}{c|}{ Ref. } & \multicolumn{1}{c|}{ Dif. } & \multicolumn{1}{c|}{ Ref. } \\
\hline Robetta & -1.63 & 70.9 & -0.02 & 0.7 & 2.73 & 68.86 \\
\hline IntFOLD4-TS & 0 & 69.27 & 0 & 0.68 & 0 & 71.6 \\
\hline RaptorX & 0.82 & 68.45 & 0 & 0.67 & 4.36 & 67.24 \\
\hline IntFOLD3-TS & 1.74 & 67.53 & 0.02 & 0.66 & 3.02 & 68.57 \\
\hline IntFOLD2-TS & 1.98 & 67.28 & 0.02 & 0.66 & 2.64 & 68.96 \\
\hline HHpredB & 2.09 & 67.17 & 0 & 0.67 & 2.59 & 69.01 \\
\hline SWISS-MODEL & 3.82 & 65.44 & 0.04 & 0.64 & 1.1 & 70.5 \\
\hline SPARKS-X & 5.26 & 64.01 & 0.03 & 0.64 & 5.54 & 66.06 \\
\hline Princeton_TEMPLATE & 9.36 & 59.91 & 0.09 & 0.59 & 15.14 & 56.46 \\
\hline NaiveBLAST & 11.57 & 57.7 & 0.12 & 0.56 & 11.15 & 60.45 \\
\hline
\end{tabular}


Table 2. Official CASP12 TBM performance comparison with Top 20 groups - All groups on 'all groups' targets. Analysis on the models designated as "1". Assessors' Formula for $\mathrm{TBM}+\mathrm{TBM} / \mathrm{FM}$ domains $\left(\mathrm{GDT} \_\mathrm{HA}+(\mathrm{SG}+\mathrm{IDDT}+\mathrm{CAD}) / 3+\mathrm{ASE}\right)$, sorted by SUM Z score $(>0.0) . *$ Denotes server groups. Data are from

http://predictioncenter.org/casp12/zscores_final.cgi.

\begin{tabular}{|c|c|c|c|c|c|c|c|c|c|c|}
\hline $\begin{array}{c}\text { GR } \\
\text { code }\end{array} \mid$ & $\begin{array}{c}\text { GR } \\
\text { name }\end{array}$ & $\begin{array}{c}\text { Domains } \\
\text { Count }\end{array}$ & $\begin{array}{c}\text { SUM } \\
\text { Zscore } \\
\text { (>-2.0) }\end{array}$ & \begin{tabular}{|c|} 
Rank \\
SUM \\
Zscore \\
$(>-2.0)$
\end{tabular} & $\begin{array}{c}\text { AVG } \\
\text { Zscore } \\
(>-2.0)\end{array}$ & \begin{tabular}{|c|} 
Rank \\
AVG \\
Zscore \\
$(>-2.0)$ \\
\end{tabular} & $\begin{array}{c}\text { SUM } \\
\text { Zscore } \\
(>0.0)\end{array}$ & \begin{tabular}{|c|} 
Rank \\
SUM \\
Zscore \\
$(>0.0)$ \\
\end{tabular} & \begin{tabular}{|c|} 
AVG \\
Zscore \\
$(>0.0)$ \\
\end{tabular} & \begin{tabular}{|c|} 
Rank \\
AVG \\
Zscore \\
$(>0.0)$ \\
\end{tabular} \\
\hline 17 & McGuffin & 29 & 27.1618 & 2 & 0.9366 & 2 & 27.882 & 1 & 0.9614 & 1 \\
\hline 417 & $\begin{array}{l}\text { VoroMQA- } \\
\text { select }\end{array}$ & 29 & 27.1702 & 1 & 0.9369 & 1 & 27.7907 & 2 & 0.9583 & 2 \\
\hline 4 & Zhang & 29 & 26.6779 & 3 & 0.9199 & 3 & 26.8982 & 3 & 0.9275 & 3 \\
\hline 203 & ProQ2 & 29 & 23.9338 & 4 & 0.8253 & 4 & 25.6378 & 4 & 0.8841 & 4 \\
\hline 73 & Wallner & 29 & 23.5157 & 5 & 0.8109 & 5 & 24.1663 & 5 & 0.8333 & 6 \\
\hline 439 & MULTICOM & 29 & 23.363 & 6 & 0.8056 & 6 & 23.839 & 6 & 0.822 & 7 \\
\hline 252 & $\begin{array}{l}\text { wfRosetta- } \\
\text { ProQ- } \\
\text { ModF6 }\end{array}$ & 28 & 19.1779 & 10 & 0.7564 & 8 & 23.6082 & 7 & 0.8432 & 5 \\
\hline 243 & Seok-refine & 29 & 20.553 & 9 & 0.7087 & 10 & 23.4151 & 8 & 0.8074 & 8 \\
\hline 479 & $\begin{array}{l}\text { Zhang- } \\
\text { Server* }\end{array}$ & 29 & 22.9713 & 7 & 0.7921 & 7 & 23.3177 & 9 & 0.8041 & 9 \\
\hline 450 & LEEab & 29 & 12.1215 & 15 & 0.418 & 17 & 22.9844 & 10 & 0.7926 & 10 \\
\hline 11 & LEE & 29 & 11.2964 & 18 & 0.3895 & 20 & 22.2071 & 11 & 0.7658 & 11 \\
\hline 183 & QUARK* & 29 & 20.8679 & 8 & 0.7196 & 9 & 21.484 & 12 & 0.7408 & 13 \\
\hline 239 & wfAll-Cheng & 27 & 14.0968 & 14 & 0.6703 & 11 & 19.5346 & 13 & 0.7235 & 14 \\
\hline 320 & raghavagps & 28 & 14.1009 & 13 & 0.575 & 13 & 19.204 & 14 & 0.6859 & 15 \\
\hline 64 & Jones-UCL & 29 & 14.4308 & 12 & 0.4976 & 15 & 17.9234 & 16 & 0.618 & 18 \\
\hline 384 & $\begin{array}{l}\text { wfMESHI- } \\
\text { Seok }\end{array}$ & 29 & 10.6692 & 19 & 0.3679 & 21 & 17.674 & 19 & 0.6094 & 20 \\
\hline 405 & IntFOLD4* & 29 & 14.6733 & 11 & 0.506 & 14 & 16.3385 & 28 & 0.5634 & 32 \\
\hline 23 & Seok & 28 & 11.6229 & 16 & 0.4865 & 16 & 16.0142 & 30 & 0.5719 & 30 \\
\hline 498 & AP_1 & 29 & 11.4258 & 17 & 0.394 & 19 & 15.9422 & 31 & 0.5497 & 34 \\
\hline 250 & $\begin{array}{l}\text { Seok- } \\
\text { server* }\end{array}$ & 29 & 10.48 & 20 & 0.3614 & 23 & 15.5036 & 34 & 0.5346 & 35 \\
\hline
\end{tabular}


Table 3. Official CASP12 TBM performance comparison with Top 20 groups - Server groups on 'all groups' + 'server only' targets. Analysis on the models designated as "1". Assessors' Formula for TBM + TBM/FM domains (GDT_HA + (SG + IDDT + CAD) $/ 3+$ ASE), sorted by SUM Z score (>-2.0). Data are from

http://predictioncenter.org/casp12/zscores_final.cgi.

\begin{tabular}{|c|c|c|c|c|c|c|c|c|c|c|}
\hline $\begin{array}{c}\text { GR } \\
\text { code }\end{array}$ & $\begin{array}{c}\text { GR } \\
\text { name }\end{array}$ & $\begin{array}{l}\text { Domain } \\
\text { s Count }\end{array}$ & $\begin{array}{l}\text { SUM } \\
\text { Zscore } \\
(>-2.0)\end{array}$ & \begin{tabular}{|c|} 
Rank \\
SUM \\
Zscor \\
$e$ \\
$(>-2.0)$
\end{tabular} & $\begin{array}{c}\text { AVG } \\
\text { Zscore } \\
(>-2.0)\end{array}$ & $\begin{array}{c}\text { Rank } \\
\text { AVG } \\
\text { Zscore } \\
(>-2.0)\end{array}$ & $\begin{array}{c}\text { SUM } \\
\text { Zscore } \\
(>0.0)\end{array}$ & $\begin{array}{c}\text { Rank } \\
\text { SUM } \\
\text { Zscore } \\
(>0.0)\end{array}$ & $\begin{array}{c}\text { AVG } \\
\text { Zscore } \\
(>0.0)\end{array}$ & $\begin{array}{c}\text { Rank } \\
\text { AVG } \\
\text { Zscore } \\
(>0.0)\end{array}$ \\
\hline 479 & \begin{tabular}{|l} 
Zhang- \\
Server
\end{tabular} & 57 & 53.2687 & 1 & 0.9345 & 1 & 53.558 & 1 & 0.9396 & 1 \\
\hline 183 & QUARK & 57 & 51.2984 & 2 & 0.9 & 2 & \begin{tabular}{|l}
51.845 \\
5
\end{tabular} & 2 & 0.9096 & 2 \\
\hline 405 & IntFOLD4 & 57 & 38.1512 & 3 & 0.6693 & 3 & \begin{tabular}{|l|}
40.700 \\
5
\end{tabular} & 5 & 0.714 & 5 \\
\hline 250 & Seok-server & 57 & 36.5368 & 4 & 0.641 & 4 & $\begin{array}{l}41.756 \\
7\end{array}$ & 4 & 0.7326 & 4 \\
\hline 236 & $\begin{array}{l}\text { MULTICOM- } \\
\text { CONSTRUC } \\
\text { T }\end{array}$ & 57 & 34.5974 & 5 & 0.607 & 5 & $\begin{array}{l}37.026 \\
2\end{array}$ & 8 & 0.6496 & 8 \\
\hline 287 & $\begin{array}{l}\text { MULTICOM- } \\
\text { CLUSTER }\end{array}$ & 57 & 34.3201 & 6 & 0.6021 & 6 & 37.661 & 7 & 0.6607 & 7 \\
\hline 345 & $\begin{array}{l}\text { MULTICOM- } \\
\text { NOVEL }\end{array}$ & 57 & 29.3235 & 7 & 0.5144 & 8 & \begin{tabular}{|l}
33.403 \\
9 \\
\end{tabular} & 9 & 0.586 & 10 \\
\hline 220 & GOAL & 57 & 24.4642 & 8 & 0.4292 & 9 & $\mid \begin{array}{l}40.020 \\
5\end{array}$ & 6 & 0.7021 & 6 \\
\hline 5 & $\begin{array}{l}\text { BAKER- } \\
\text { ROSETTAS } \\
\text { ERVER }\end{array}$ & 57 & 22.6251 & 9 & 0.3969 & 10 & $\begin{array}{l}43.556 \\
7\end{array}$ & 3 & 0.7642 & 3 \\
\hline 119 & HHPred0 & 57 & 10.6627 & 10 & 0.1871 & 11 & $\begin{array}{l}21.332 \\
2\end{array}$ & 14 & 0.3742 & 17 \\
\hline 349 & HHPred1 & 57 & 10.2547 & 11 & 0.1799 & 12 & 21.102 & 15 & 0.3702 & 18 \\
\hline 251 & $\begin{array}{l}\text { myprotein- } \\
\text { me }\end{array}$ & 57 & 7.6733 & 12 & 0.1346 & 13 & $\begin{array}{l}25.049 \\
8\end{array}$ & 12 & 0.4395 & 13 \\
\hline 48 & $\begin{array}{l}\text { ToyPred_em } \\
\text { ail }\end{array}$ & 57 & 6.0518 & 13 & 0.1062 & 14 & $\begin{array}{l}27.666 \\
9\end{array}$ & 10 & 0.4854 & 11 \\
\hline 92 & RaptorX & 57 & 5.921 & 14 & 0.1039 & 15 & \begin{tabular}{|l|}
27.623 \\
8 \\
\end{tabular} & 11 & 0.4846 & 12 \\
\hline 313 & HHGG & 57 & 4.1865 & 15 & 0.0734 & 16 & \begin{tabular}{|l}
24.656 \\
9
\end{tabular} & 13 & 0.4326 & 14 \\
\hline 425 & $\begin{array}{l}\text { FALCON_T } \\
\text { OPOX }\end{array}$ & 57 & -5.3579 & 16 & -0.094 & 18 & $\begin{array}{l}16.000 \\
2 \\
\end{array}$ & 21 & 0.2807 & 23 \\
\hline 77 & FALCON_T & 57 & -5.3673 & 17 & -0.0942 & 19 & 16.483 & 19 & 0.2892 & 21 \\
\hline
\end{tabular}




\begin{tabular}{|l|l|l|l|l|l|l|l|l|l|l|}
\hline & OPO & & & & & & 2 & & & \\
\hline 16 & FFAS-3D & 57 & -7.8423 & 18 & -0.1376 & 21 & $\begin{array}{l}14.376 \\
6\end{array}$ & 23 & 0.2522 & 26 \\
\hline 446 & YASARA & 56 & -8.1083 & 19 & -0.1091 & 20 & $\begin{array}{l}20.585 \\
9\end{array}$ & 17 & 0.3676 & 19 \\
\hline 464 & tsspred2 & 57 & -10.2517 & 20 & -0.1799 & 22 & 12.695 & 27 & 0.2227 & 30 \\
\hline
\end{tabular}


Table 4. Comparative performance of the single-template and multiple-template modelling stages of the IntFOLD4-TS server pipeline. GDT_TS scores of the top models selected for the TBM + TBM/FM domains. IntFOLD60 \& IntFOLD140 refer to the top models selected by ModFOLDclust2 from the datasets shown in Figure 1. LOMETS, LOMETS version 4.4. IT4, top model from I-TASSER version 4.4 with default parameters in light mode $(<=5 \mathrm{~h}$ walltime). HHpred, top HHpred model from HHsuite version 3.0.0. IntFOLD3, top model selected from the final pool of models using the ModFOLDclust approach used by IntFOLD3-TS. The row labelled "t-test v IntFOLD4" shows the p-value for the paired samples t-tests for the scores from the method in the column versus those from the IntFOLD4 column. *The IT4 method was not run for targets $>600$ residues, so for T0912 the top LOMETS model score is used.

\begin{tabular}{|c|c|r|r|r|r|r|r|r|}
\hline \multicolumn{2}{|c|}{} & \multicolumn{3}{|c|}{ Single-template methods } & \multicolumn{3}{c|}{ Multiple-template methods } \\
\hline $\begin{array}{c}\text { Domain } \\
\text { class }\end{array}$ & Target & IntFOLD60 & IntFOLD140 & LOMETS & \multicolumn{1}{c|}{ IT4 } & HHpred & IntFOLD3 & IntFOLD4 \\
\hline FM/TBM & T0868-D1 & 0.2220 & 0.1659 & 0.1681 & 0.3427 & 0.2457 & 0.2522 & 0.1918 \\
\hline FM/TBM & T0884-D1 & 0.2148 & 0.2324 & 0.2500 & 0.2641 & 0.1901 & 0.2535 & 0.3134 \\
\hline FM/TBM & T0890-D1 & 0.3963 & 0.3415 & 0.3079 & 0.3384 & 0.1890 & 0.3384 & 0.3415 \\
\hline FM/TBM & T0892-D1 & 0.0833 & 0.4384 & 0.2862 & 0.4928 & 0.3587 & 0.4348 & 0.4928 \\
\hline FM/TBM & T0894-D2 & 0.1296 & 0.1343 & 0.1481 & 0.1759 & 0.2315 & 0.1296 & 0.3611 \\
\hline FM/TBM & T0896-D1 & 0.0843 & 0.0988 & 0.0000 & 0.0930 & 0.2384 & 0.0988 & 0.0988 \\
\hline FM/TBM & T0896-D2 & 0.3488 & 0.3200 & 0.2888 & 0.0588 & 0.1300 & 0.3250 & 0.3238 \\
\hline FM/TBM & T0898-D2 & 0.2000 & 0.1045 & 0.2273 & 0.3045 & 0.4500 & 0.3955 & 0.3045 \\
\hline FM/TBM & T0901-D1 & 0.2937 & 0.2511 & 0.2500 & 0.2433 & 0.2478 & 0.2444 & 0.2489 \\
\hline FM/TBM & T0909-D1 & 0.2793 & 0.3026 & 0.0323 & 0.2605 & 0.2905 & 0.2905 & 0.2905 \\
\hline FM/TBM & T0912-D2 & 0.1205 & 0.3313 & 0.3223 & 0.3223 & 0.3313 & 0.3373 & 0.3223 \\
\hline FM/TBM & T0943-D1 & 0.1290 & 0.0000 & 0.0000 & 0.1935 & 0.3347 & 0.1250 & 0.3105 \\
\hline FM/TBM & T0945-D1 & 0.0660 & 0.2627 & 0.2400 & 0.2487 & 0.3507 & 0.2387 & 0.2487 \\
\hline TBM & T0860-D1 & 0.0735 & 0.5239 & 0.5276 & 0.5184 & 0.4724 & 0.5092 & 0.5184 \\
\hline TBM & T0861-D1 & 0.7965 & 0.7965 & 0.8277 & 0.7837 & 0.8365 & 0.8638 & 0.8269 \\
\hline TBM & T0865-D1 & 0.4073 & 0.7137 & 0.7137 & 0.3589 & 0.5645 & 0.5403 & 0.5645 \\
\hline TBM & T0867-D1 & 0.8918 & 0.8654 & 0.8149 & 0.6611 & 0.8774 & 0.8726 & 0.8798 \\
\hline
\end{tabular}




\begin{tabular}{|c|c|c|c|c|c|c|c|c|}
\hline TBM & T0872-D1 & 0.4205 & 0.4063 & 0.4261 & 0.4716 & 0.4063 & 0.4261 & 0.4886 \\
\hline TBM & T0877-D1 & 0.4384 & 0.4384 & 0.3908 & 0.4225 & 0.4366 & 0.4437 & 0.4296 \\
\hline TBM & T0879-D1 & 0.5489 & 0.5489 & 0.4693 & 0.4727 & 0.5398 & 0.5784 & 0.5784 \\
\hline TBM & T0881-D1 & 0.4047 & 0.4047 & 0.3861 & 0.3354 & 0.4703 & 0.4245 & 0.4059 \\
\hline TBM & T0882-D1 & 0.3766 & 0.3766 & 0.5380 & 0.4272 & 0.5759 & 0.3703 & 0.5063 \\
\hline TBM & T0885-D1 & 0.3728 & 0.4276 & 0.2632 & 0.4912 & 0.3553 & 0.4737 & 0.5307 \\
\hline TBM & T0889-D1 & 0.6715 & 0.6715 & 0.6130 & 0.5962 & 0.6893 & 0.6611 & 0.6674 \\
\hline TBM & T0891-D1 & 0.6875 & 0.6875 & 0.7031 & 0.6853 & 0.7232 & 0.7165 & 0.6920 \\
\hline TBM & T0893-D1 & 0.4418 & 0.4418 & 0.3048 & 0.3048 & 0.3322 & 0.2979 & 0.4110 \\
\hline TBM & T0893-D2 & 0.5621 & 0.5621 & 0.5695 & 0.5636 & 0.6568 & 0.5843 & 0.5932 \\
\hline TBM & T0895-D1 & 0.1146 & 0.4458 & 0.4979 & 0.4500 & 0.5063 & 0.4771 & 0.4750 \\
\hline TBM & T0902-D1 & 0.3604 & 0.3139 & 0.3139 & 0.3268 & 0.3766 & 0.3160 & 0.2955 \\
\hline TBM & T0903-D1 & 0.6759 & 0.7330 & 0.7130 & 0.7130 & 0.6512 & 0.7461 & 0.7461 \\
\hline TBM & T0912-D1 & 0.2530 & 0.3804 & 0.2512 & $0.2512^{*}$ & 0.2729 & 0.2699 & 0.2633 \\
\hline TBM & T0913-D1 & 0.3506 & 0.3698 & 0.3698 & 0.3491 & 0.3913 & 0.3772 & 0.3772 \\
\hline TBM & T0917-D1 & 0.5185 & 0.5185 & 0.4955 & 0.5192 & 0.5492 & 0.5301 & 0.5301 \\
\hline TBM & T0920-D1 & 0.4346 & 0.4525 & 0.4206 & 0.4136 & 0.4829 & 0.4470 & 0.4813 \\
\hline TBM & T0920-D2 & 0.0365 & 0.0411 & 0.0103 & 0.0491 & 0.3824 & 0.0411 & 0.0434 \\
\hline TBM & T0921-D1 & 0.4112 & 0.3895 & 0.4112 & 0.4330 & 0.4076 & 0.4565 & 0.4112 \\
\hline TBM & T0922-D1 & 0.5135 & 0.5135 & 0.5034 & 0.5034 & 0.5439 & 0.5980 & 0.5980 \\
\hline TBM & T0928-D1 & 0.2610 & 0.2559 & 0.2617 & 0.2683 & 0.3028 & 0.2808 & 174 \\
\hline TBM & T0942-D1 & 0.1040 & 0.1171 & 0.5650 & 0.1315 & 0.6012 & 0.6012 & 0.5997 \\
\hline TBM & T0942-D2 & 0.2710 & 0.2757 & 0.0572 & 0.2757 & 0.3435 & 0.3435 & 0.3353 \\
\hline TBM & T0943-D2 & 0.2886 & 0.2897 & 0.2897 & 0.2757 & 0.3809 & 0.2349 & 0.2942 \\
\hline TBM & T0944-D1 & 0.4792 & 0.4792 & 0.4101 & 0.3676 & 0.4881 & 0.5089 & 0.5119 \\
\hline TBM & T0946-D2 & 0.3573 & 0.3679 & 0.2217 & 0.2476 & 0.1769 & 0.3125 & 0.3172 \\
\hline TBM & T0947-D1 & 0.3600 & 0.3600 & 0.3929 & 0.3800 & 0.3786 & 0.4100 & 0.3800 \\
\hline \multirow[t]{3}{*}{ TBM } & T0948-D1 & 0.1493 & 0.5084 & 0.4765 & 0.4715 & 0.5017 & 0.4832 & 0.5101 \\
\hline & Total & 15.4516 & 17.1520 & 16.2540 & 16.3858 & 18.7612 & 18.1769 & 18.9180 \\
\hline & $\begin{array}{l}\text { t-test v } \\
\text { IntFOLD4 }\end{array}$ & 7.93E-05 & $6.59 \mathrm{E}-03$ & $3.14 \mathrm{E}-05$ & $1.15 \mathrm{E}-04$ & 3.97E-01 & $2.66 \mathrm{E}-02$ & \\
\hline
\end{tabular}

